\title{
Metastases of Melanoma to Head and Neck Mucosa: A Report of Short Series
}

\author{
Raphaela Silva Leandro Santos ${ }^{1}$ Marília Ferreira Andrade ${ }^{2,3} \cdot$ Fábio de Abreu Alves ${ }^{2,3} \cdot$ Luiz Paulo Kowalski²,3 \\ Danyel Elias da Cruz Perez ${ }^{1}$
}

${ }^{I}$ Department of Clinical and Preventive Dentistry, Oral Pathology Section, School of Dentistry, Federal University of Pernambuco, Recife;

Departments of ${ }^{2}$ Stomatology and ${ }^{3}$ Otorhinolaryngology-Head and Neck Surgery, A. C. Camargo Cancer Center, São Paulo, Brazil

\begin{abstract}
Objectives. Metastasis of melanoma to the head and neck mucosa is a very unusual condition. The aim of this study was to report four cases of patients with metastatic melanoma in the head and neck mucosa treated at a single institution.

Methods. Clinical data were obtained from the medical records. All cases were histologically reviewed to confirm the diagnosis, and immunohistochemical reactions were performed in the cases submitted to biopsy.

Results. All patients were males and the mean age was 40.5 years old. The sites of the metastatic tumors were gingival mucosa, floor of the mouth, oropharynx, and larynx. Two tumors appeared as submucosal nodules with normal color; one lesion was a blackish nodular lesion, and one was shown to be an ulcerated lesion. The size of tumors ranged from 2.0 to $4.0 \mathrm{~cm}$. All patients had developed systemic disease at time of diagnosis of metastatic tumor in the head and neck mucosa. Survival rates ranged from 2 to 19 months after the diagnosis of the metastatic mucosal melanoma in the head and neck region.

Conclusion. Although rare, patients with melanoma must be closely and regularly followed up, with careful routine examination of head and neck, because metastatic tumors in this region seem to be part of a lethal widespread metastatic disease.
\end{abstract}

Keywords. Head and Neck Neoplasms; Melanoma; Mouth; Mucous Membrane; Neoplasm Metastasis

\section{INTRODUCTION}

Malignant mucosal melanoma is a rare tumor. It appears as a very aggressive neoplasm and arises mainly in mucous membranes of the head and neck region, female genital organs, and anorectal or urinary tracts. Over $50 \%$ of all mucosal melanomas occur in the mucosa of the head and neck region, arising mainly in the upper digestive tract and oral cavity [1,2].

The mucosa of the head and neck region, including the oral cavity, may also be affected by metastatic melanomas. Metastases of melanomas to this region are very rare. In spite of most patients usually presenting several metastatic tumors at diagnosis, these lesions may represent a diagnostic challenge. There are very few previously published studies with series of cases of metastases of the melanoma to the head and neck mucosa [3-7]. Thus, the aim of this study was to report 4 cases of metastatic melanoma to the mucosal surfaces of the head and neck.

\section{MATERIALS AND METHODS}

Between 1980 and 2010, the records of the patients with metastases of melanoma located in the head and neck mucosa, obtained from the archives of the Departments of Stomatology and Head and Neck Surgery and Otorhinolaryngology, A.C. Camargo Cancer Center, Sao Paulo, Brazil, were selected for the 
study. Clinical data such as age, gender, site of the metastases, site of the primary tumor, time between the initial diagnosis and development of metastases, treatment and outcome were obtained from the medical records.

All cases were histologically reviewed. In order to confirm the diagnosis, immunohistochemical reactions against S-100 protein (polyclonal, dilution 1:5,000; Sigma Aldrich Co., St. Louis, MO, USA) and HMB45 (HMB45 clone, dilution 1:400; Dako, Glostrup, Denmark) were performed in the cases that were submitted to incisional biopsy or other surgical procedures. Antigen retrieval was performed in a pressure cooker for 4 minutes using a 10mmol L ${ }^{-1}$ citrate buffer ( $\mathrm{pH}$ 6.0). Incubations with the primary antibody were performed for 18 hours at $4^{\circ} \mathrm{C}$. After this, the tissue sections were incubated with Post Primary Block for 30 minutes at $37^{\circ} \mathrm{C}$ (NovoLink Max Polymer, Novocastra, Newcastle, UK), followed by application of diaminobendizine as the chromogen. Slides were counterstained with Carazzi hematoxylin. The study has been approved by the Institutional Review Board of the Federal University of Pernambuco (protocol number: 44536715.8.0000.5208).

\section{RESULTS}

The clinical outcome and immunohistochemical features are summarized in the Table 1 . All patients were males and presented similar age $(36,39,43$, and 44 years old). The site of the metastatic tumors was the gingival mucosa, floor of mouth, oropharynx, and larynx. In cases 1 and 3, the lesions appeared as submucosal nodules with normal color, whereas in case 2 , it presented as a blackish nodular lesion (Fig. 1). An ulcerated lesion presented in case 4. All patients complained of pain and the patient with the laryngeal tumor also complained of hoarseness (case 4). Mean size of the tumors was $3.0 \mathrm{~cm}$ (range, 2.0 to 4.0 $\mathrm{cm}$ ) and the metastatic tumors arose after a mean time of 25.2 months (range, 8 to 48 months) after the initial diagnosis of melanoma. Primary tumor sites were in the skin (cases 1 and 4) and two cases presented occult primary tumors (cases 2 and 3). These 2 cases were initially diagnosed because of neck lymph nodes metastases. The diagnostic workup failed to reveal the primary tumor. In these 2 cases, when the metastatic mucosal melanoma was diagnosed, the patients had already shown metastases in other distant organs. In the same way, the other cases (cases 1 and 4) also presented other distant metastases at diagnosis of the metastases in the head and neck mucosa (Table 1).

In all cases but one (case 2), an incisional biopsy was performed to confirm the diagnosis. Microscopically, the lesions showed spreading amelanotic tumor cells with atypical nuclei and mitoses, in addition to evident cellular pleomorphism. Furthermore, the epithelium covering the lesions was intact and atrophic, without junctional activity (Fig. 2). The tumor cells from case 4 presented discrete pigmentation by melanin. All microscopically available cases were positive for S-100 protein (Fig. 3A) and only one case (case 3) was positive for HMB45 (Fig. 3B).

All cases were treated by systemic chemotherapy using CVD (cisplatin/vinblastine/dacarbazine) and taxol weekly. In addition, immunotherapeutic agents interleukin-2 (IL-2) and interferon-alpha (INF- $\alpha$ ) were used in the case of metastasis to the floor of the mouth. The case with a metastatic tumor located in the larynx was also submitted to laser partial laryngectomy to open the airways and avoid tracheotomy. All patients died of the disease and survival ranged from 2 to 19 months after the diagnosis of metastatic melanoma in head and neck mucosa.

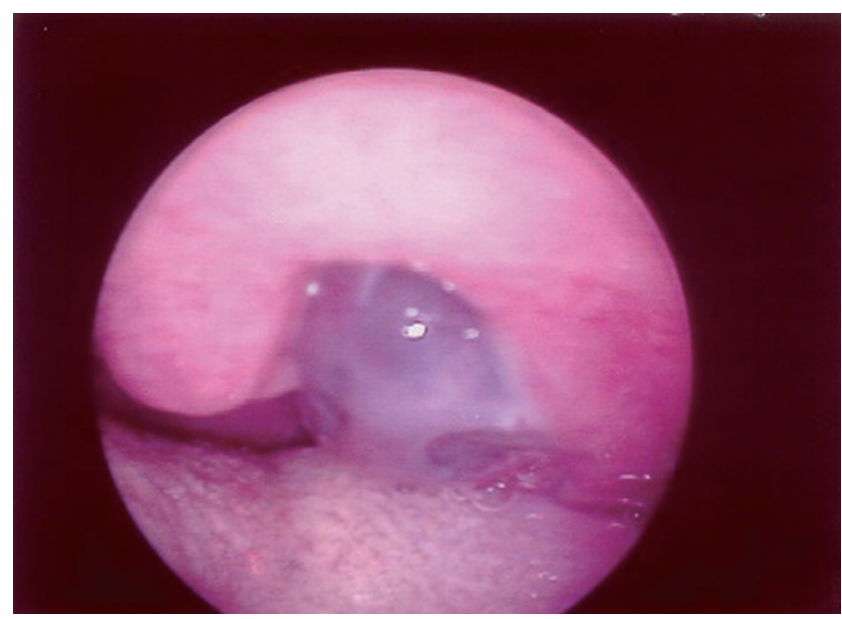

Fig. 1. Case 2 appearing as a blackish submucosal nodule located on the oropharynx.

Table 1. Clinical, outcome and immunohistochemical features of cases of metastatic mucosal melanoma to head and neck

\begin{tabular}{|c|c|c|c|c|c|c|c|c|}
\hline Case & $\begin{array}{l}\text { Age } \\
(y r)\end{array}$ & $\begin{array}{c}\text { Site of metastatic } \\
\text { tumor }\end{array}$ & $\begin{array}{l}\text { Size } \\
(\mathrm{cm})\end{array}$ & Primary site & $\begin{array}{l}\text { Time between diagnosis } \\
\text { of primary tumor and } \\
\text { metastasis (mo) }\end{array}$ & Other metastatic sites & Immunohistochemical & $\begin{array}{l}\text { Survival time } \\
\qquad(\mathrm{mo})\end{array}$ \\
\hline 1 & 44 & Gingiva & 2.0 & Chest skin & 8 & Generalized metastases & S-100 protein: +/HMB45:- & 2 \\
\hline 2 & 36 & Oropharynx & 4.0 & Unknown & 21 & Lung, brain, and intestine & NA & 5 \\
\hline 3 & 43 & Floor of the mouth & 4.0 & Unknown & 24 & $\begin{array}{l}\text { Lung, brain, and axillaries } \\
\text { lymph nodes }\end{array}$ & S-100 protein: +/HMB45: + & 19 \\
\hline 4 & 39 & Larynx & 2.0 & Axillary skin & 48 & $\begin{array}{l}\text { Lung, subcutaneous, } \\
\text { liver, and pericardium }\end{array}$ & S-100 protein: +/HMB45:- & 14 \\
\hline
\end{tabular}

NA, not available. 


\section{DISCUSSION}

Only $0.6 \%$ to $9.3 \%$ of patients with cutaneous melanoma will develop metastases in the mucosa of the upper aerodigestive

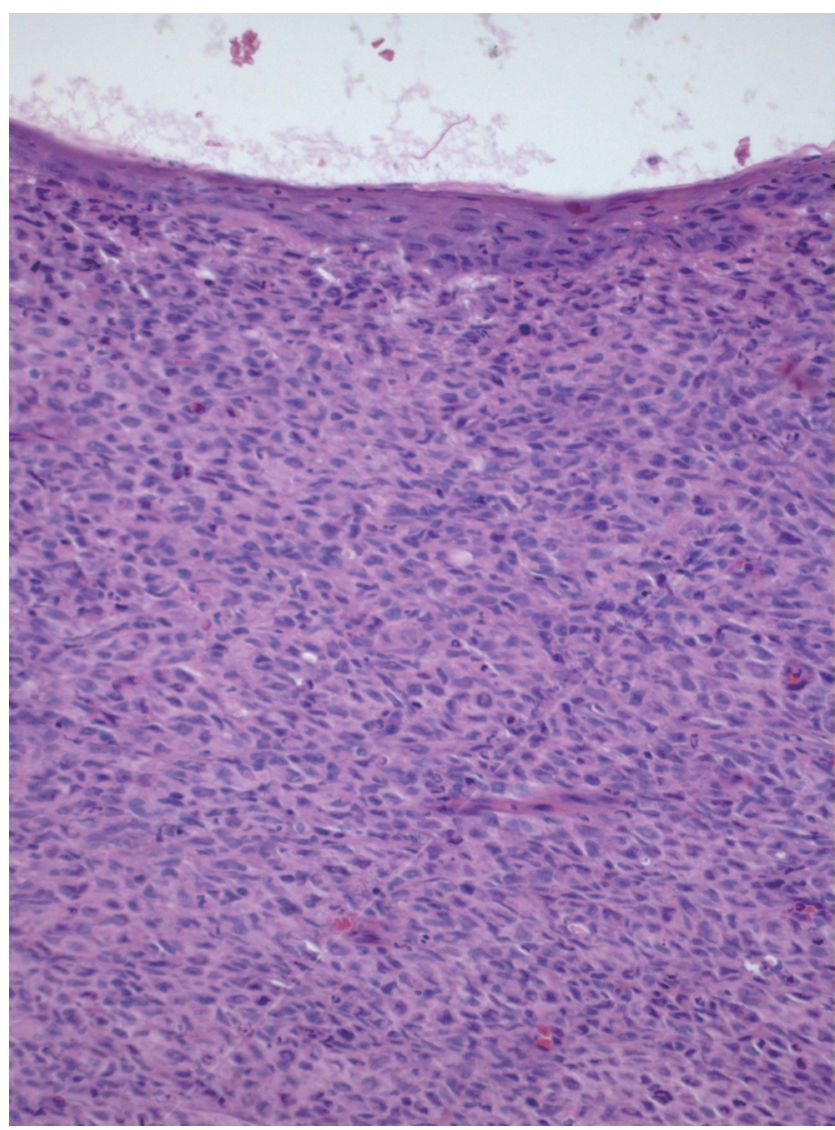

Fig. 2. Case 1: Spindle and epithelioid malignant cells adjacent to intact and atrophic squamous epithelium that recovered the oral mucosa. Melanin was not observed (H\&E, $\times 200)$.

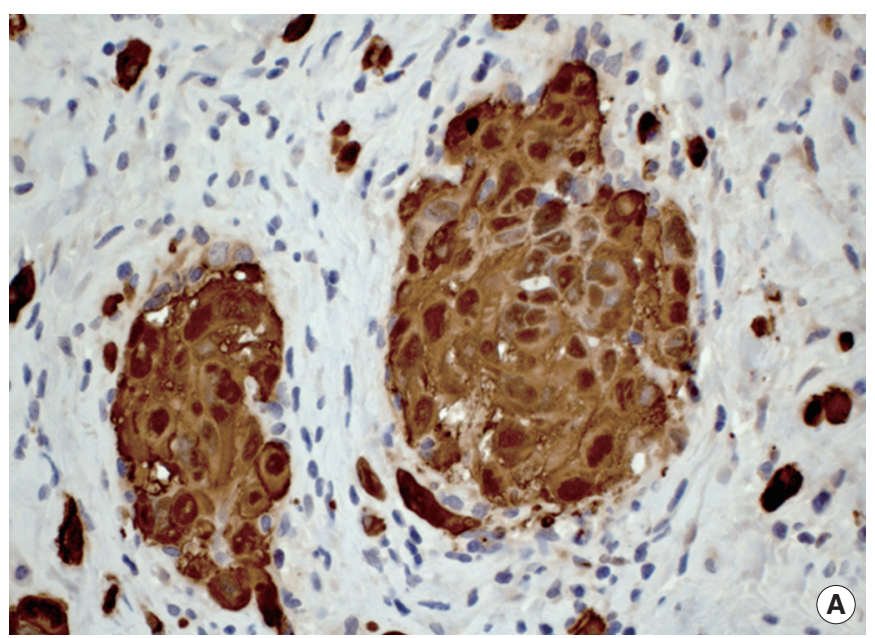

tract. There are few discussions in the literature about this condition $[3,4,6,7]$. Metastases of melanoma to head and neck mucosa, including the oral cavity, are very rare $[3,5,6]$. Considering the frequency of oral metastases, these tumors represent approximately $1 \%$ of all oral tumor lesions, and are usually evidence of a widespread disease. In the oral cavity, bone metastases are more common than soft tissue metastases, and the major primary sites presenting oral metastases are the lung, kidney, liver, and prostate for men, and breast, female genital organs, kidney, and colo-rectum for women [8]. Of the cases presented in the current series, two affected the oral mucosa.

Some studies have found no difference in incidence relative to gender when the metastatic melanomas to the head and neck region were considered $[4,9]$. In our study all patients were males. In the present series, the mean age of the patients at the time of diagnosis of the mucosal metastases was 40.5 years old, which is lower than the mean age found by other authors [9]. As regards the most common site of occurrence, primary and metastatic melanomas of the head and neck region present different features. The most frequent site for primary mucosal melanoma in the upper respiratory tract is the nasal cavity, followed by paranasal sinuses, oral cavity, pharynx, larynx, and esophagus $[4,10]$. In the oral cavity, almost $80 \%$ of all primary melanomas occur in the keratinized mucosa of the hard palate, followed by maxillary and mandibular, and buccal mucosa [1]. Metastatic melanomas of the head and neck mucosa have been found most often in the oropharynx, larynx, oral cavity, nasopharynx, tonsil and lip $[3,4,7]$. Our study reports two cases of metastatic melanoma in uncommon sites of the oral cavity (floor of mouth and gingival mucosa), one case in the oropharynx and another in the larynx.

The clinical features of metastatic melanoma involving the upper aerodigestive tract include pain, bleeding, airway obstruction and presence of mass or ulceration $[3,4]$. In our series, the patients complained of pain and the metastatic tumors appeared

Fig. 3. (A) Case 4: Tumor cells showing strong positivity for S-100 protein (immunohistochemistry, $\times 400$ ). (B) Case 3: Pleomorphic malignant cells presenting cytoplasmic positivity for HMB45 (immunohistochemistry, $\times 400$ ). 
as submucosal nodules and ulcerated lesions. Airway obstruction was also observed in the tumor situated in the larynx.

Primary and secondary melanomas of the upper aero digestive tract mucosa mimic each other clinically and require microscopic examination in order to differentiate them. The histopathological diagnosis of primary melanoma requires the presence of junctional activity in the overlying or adjacent lateral mucosa or both, and epidermal migration may be also observed. In contrast, metastatic melanoma is typically covered by an unrated mucosal layer [3,4]. A metastatic tumor must have both intact overlying mucosa and adjacent mucosa devoid of junctional changes, as observed in our cases.

On immunohistochemical analysis, melanomas react positively to antibodies HMB45, S-100 protein and Melan-A [1]. In the three cases in which incisional biopsy was performed, the tumors were positive for S-100 and one of them was also positive for HMB45, which confirmed the diagnosis of melanoma.

Conventional treatments for melanoma include surgery, chemotherapy, and immunotherapy; of these, surgery provides optimum results for patients at all stages of the disease. Unfortunately, for those patients presenting with metastases, disseminated tumors in distant organs are often unresectable and until now, very few therapeutic options have been available to these patients [11]. In metastatic tumors, surgery is recommended only in exceptional cases for palliative purposes [12] such as in the laryngeal metastatic melanoma of the present series, which was submitted to laser partial laryngectomy to open the airways.

Melanoma is resistant to many forms of traditional chemotherapy. A large number of clinical trials have tested different single drug-like alkylating agents, nitrosoureas, vinca alkaloids, platinum drugs, taxanes, topoisomerase inhibitors, and anthracyclines, but few have shown an objective response rate $(<20 \%)$ or an increase in progression-free and overall survival rates [13]. Dacarbazine is the conventional therapy for metastatic melanomas at this time, although the response rate is only $14 \%$ to $20 \%$. The mean response duration is 4 to 6 months and fewer than $2 \%$ of patients survive 6 years [1]. The disappointing results with single agent chemotherapy has led to the evaluation of multidrug combination regimens in efforts to improve the outcome and enhance response rates in patients with metastatic disease. In phase II trials, two of the most effective combinations (that produced response rates ranging from $30 \%$ to $50 \%$ ) were the three-drug combination of CVD and the four-drug combination (Dartmouth combination) of CDBT (cisplatin/dacarbazine/carmustine and tamoxifen). Although in randomized phase III trials no differences were shown in either response duration or survival [1,13]. All our patients were treated with systemic chemotherapy using CVD and taxol weekly.

Spontaneous tumor regression in patients with metastatic melanoma have been reported. These cases suggested that immunotherapy might have a higher impact on the outcome of metastatic melanoma than in other cancers. Therapies with biologic re- sponse modifiers (cytokines) especially IL-2 and INF- $\alpha$ have important roles in both adjuvant therapy and treatment of metastatic melanoma [13]. Treatment with the combination of biologic therapies and chemotherapeutic agents has been performed in patients who have advanced melanoma, including those who have mucosal melanoma of the head and neck, with some limited success [10]. In our study, immunotherapeutic agents (IL-2 and INF- $\alpha$ ) were used in the case of metastasis to the floor of the mouth. These agents were used in combination with chemotherapy in an endeavor to improve the treatment response.

Since the molecular mechanisms of melanoma cell survival have been unravelled, several promising approaches to the treatment of metastatic disease have emerged. Nanomedicine holds the promise of revolutionizing the treatment of patients with cancer in the future. Several nanoparticles are being engineered and tested for tumor imaging and targeted drug delivery for melanoma. Generally, the encapsulation of drugs in nanoparticles has been reported to improve the pharmacokinetic properties of the compounds because of the better tumor accumulation by passive and active targeting, sustained drug release, longer blood circulation times, and the incorporation of nanoparticles into cells. This increases the efficacy of treatment, especially in advanced cases. In metastatic melanoma, although every tissue has its own unique challenges to nanoparticle penetration, for many solid tumors, nanoparticle delivery through intravenous administration is promoted by the enhanced permeability and retention effect, due to the abnormal vascular architecture and insufficient lymphatic drainage in tumors [11].

The prognosis for patients with metastatic melanoma of mucosal surfaces of the head and neck is poor, since the disease has a very aggressive behavior. Usually, the patients had already developed systemic disease at the time when the metastasis was diagnosed [3,4,7]. Advanced melanoma is associated with an extremely poor median survival, ranging from 2 to 8 months [13]. In the current series, all patients had systemic disease at the time of being diagnosed with metastatic mucosal tumor.

Metastatic melanoma of unknown primary origin accounts for approximately $2 \%-3 \%$ of all melanoma cases $[14,15]$. The prognostic significance of metastases of primary known tumors and primary unknown tumors is still controversial [16]. In metastatic and primary tumors with the same clinical stages, several studies have shown that survival rates are similar [14-17]. In this series, when primary tumors were unknown, the diagnosis was based on positive neck lymph nodes. The primary origin of melanoma in lymph nodes is one hypothesis for an unknown primary melanoma appearing in lymph nodes. Proponents of this theory suggest that the reason why the prognosis after surgery is similar in this patient group with known and unknown primary lesions is because the occult primary tumor is removed with the nodal dissection [18]. In the cases presented, despite the fact that all patients died, we observed that patients with a known primary tumor (gingival mucosa and oropharynx metastases) 
died some months after the diagnosis of their metastasis; while the patients whose primary tumor location was unknown (larynx and mouth floor metastases) died after 2.5 years and 2.2 years respectively after the diagnosis. Others studies have also found a better survival rate for patients with metastasis of unknown primary tumors [19-21].

In summary, in spite of the improvement of the treatment modalities, the prognosis of metastatic melanomas remains unfavorable, since that these tumors seem to be part of a lethal widespread metastatic disease. Site of the primary tumor does not seem be a predictive factor, because the metastatic pattern of malignant melanoma is variable. Early diagnosis of primary tumors provides greater chances of successful treatment. Although rare, patients with melanoma must be closely and regularly followed-up with careful routine examination of head and neck.

\section{CONFLICT OF INTEREST}

No potential conflict of interest relevant to this article was reported.

\section{REFERENCES}

1. Lengyel E, Gilde K, Remenar E, Esik O. Malignant mucosal melanoma of the head and neck. Pathol Oncol Res. 2003;9(1):7-12.

2. Patrick RJ, Fenske NA, Messina JL. Primary mucosal melanoma. J Am Acad Dermatol. 2007 May;56(5):828-34.

3. Henderson LT, Robbins KT, Weitzner S. Upper aerodigestive tract metastases in disseminated malignant melanoma. Arch Otolaryngol Head Neck Surg. 1986 Jun;112(6):659-63.

4. Billings KR, Wang MB, Sercarz JA, Fu YS. Clinical and pathologic distinction between primary and metastatic mucosal melanoma of the head and neck. Otolaryngol Head Neck Surg. 1995 Jun;112(6): 700-6.

5. Aydogan LB, Myers JN, Myers EN, Kirkwood J. Malignant melanoma metastatic to the tonsil. Laryngoscope. 1996 Mar;106(3 Pt 1): 313-6.

6. Coehlo FH, Pellicioli AC, Martins MA, Pavesi VC, Schmerling RA, Martins MD. Multiple metastases of malignant melanoma in the head and neck: a case report and literature review. Gen Dent. 2014 Jan-Feb;62(1):43-8.

7. Mifsud M, Padhya TA. Metastatic melanoma to the upper aerodigestive tract: a systematic review of the literature. Laryngoscope. 2014
May;124(5):1143-9.

8. Hirshberg A, Shnaiderman-Shapiro A, Kaplan I, Berger R. Metastatic tumours to the oral cavity: pathogenesis and analysis of 673 cases. Oral Oncol. 2008 Aug;44(8):743-52.

9. Khan KH, Goody RB, Hameed H, Jalil A, Coyle VM, McAleer JJ. Metastatic melanoma: a regional review and future directions. Tumori. 2012 Sep-Oct;98(5):575-80.

10. Larson DL, Larson JD. Head and neck melanoma. Clin Plast Surg. 2010 Jan;37(1):73-7.

11. Bombelli FB, Webster CA, Moncrieff M, Sherwood V. The scope of nanoparticle therapies for future metastatic melanoma treatment. Lancet Oncol. 2014 Jan;15(1):e22-32.

12. Morgan AH, Norris JW, Hicks JN. Palliative laser surgery for melanoma metastatic to the larynx: report of two cases. Laryngoscope. 1985 Jul;95(7 Pt 1):794-7.

13. Mouawad R, Sebert M, Michels J, Bloch J, Spano JP, Khayat D. Treatment for metastatic malignant melanoma: old drugs and new strategies. Crit Rev Oncol Hematol. 2010 Apr;74(1):27-39.

14. Katz KA, Jonasch E, Hodi FS, Soiffer R, Kwitkiwski K, Sober AJ, et al. Melanoma of unknown primary: experience at Massachusetts General Hospital and Dana-Farber Cancer Institute. Melanoma Res. 2005 Feb;15(1):77-82.

15. de Waal AC, Aben KK, van Rossum MM, Kiemeney LA. Melanoma of unknown primary origin: a population-based study in the Netherlands. Eur J Cancer. 2013 Feb;49(3):676-83.

16. Schlagenhauff B, StroebelW, Ellwanger U, Meier F, Zimmermann C, Breuninger $\mathrm{H}$, et al. Metastatic melanoma of unknown primary origin shows prognostic similarities to regional metastatic melanoma: recommendations for initial staging examinations. Cancer. $1997 \mathrm{Jul}$ 1;80(1):60-5.

17. Kamposioras K, Pentheroudakis G, Pectasides D, Pavlidis N. Malignant melanoma of unknown primary site. To make the long story short.A systematic review of the literature. Crit Rev Oncol Hematol. 2011 May;78(2):112-26.

18. O’Neill JK, Khundar R, Knowles L, Scott-Young N, Orlando A. Melanoma with an unknown primary: a case series. J Plast Reconstr Aesthet Surg. 2010 Dec;63(12):2071-80.

19. Wang BY, Lawson W, Robinson RA, Perez-Ordonez B, Brandwein M. Malignant melanomas of the parotid: comparison of survival for patients with metastases from known vs unknown primary tumor sites. Arch Otolaryngol Head Neck Surg. 1999 Jun;125(6):635-9.

20. Prens SP, van der Ploeg AP, van Akkooi AC, van Montfort CA, van Geel AN, de Wilt JH, et al. Outcome after therapeutic lymph node dissection in patients with unknown primary melanoma site. Ann Surg Oncol. 2011 Dec;18(13):3586-92.

21. Weide B, Faller C, Elsasser M, Buttner P, Pflugfelder A, Leiter U, et al. Melanoma patients with unknown primary site or nodal recurrence after initial diagnosis have a favourable survival compared to those with synchronous lymph node metastasis and primary tumour. PLoS One. 2013 Jun 25;8(6):e66953. 